\title{
POSITIONAL DETERMINACY OF GAMES WITH INFINITELY MANY PRIORITIES *
}

\author{
ERICH GRÄDEL ${ }^{a}$ AND IGOR WALUKIEWICZ ${ }^{b}$ \\ ${ }^{a}$ Mathematische Grundlagen der Informatik, RWTH Aachen University, D-52056 Aachen, Germany \\ e-mail address: graedel@informatik.rwth-aachen.de \\ ${ }^{b}$ LaBRI , Université Bordeaux-1, 351 Cours de la Libération, 33405 Talence, France \\ e-mail address: igw@labri.fr
}

\begin{abstract}
We study two-player games of infinite duration that are played on finite or infinite game graphs. A winning strategy for such a game is positional if it only depends on the current position, and not on the history of the play. A game is positionally determined if, from each position, one of the two players has a positional winning strategy.

The theory of such games is well studied for winning conditions that are defined in terms of a mapping that assigns to each position a priority from a finite set $C$. Specifically, in Muller games the winner of a play is determined by the set of those priorities that have been seen infinitely often; an important special case are parity games where the least (or greatest) priority occurring infinitely often determines the winner. It is well-known that parity games are positionally determined whereas Muller games are determined via finite-memory strategies.

In this paper, we extend this theory to the case of games with infinitely many priorities. Such games arise in several application areas, for instance in pushdown games with winning conditions depending on stack contents.

For parity games there are several generalisations to the case of infinitely many priorities. While max-parity games over $\omega$ or min-parity games over larger ordinals than $\omega$ require strategies with infinite memory, we can prove that min-parity games with priorities in $\omega$ are positionally determined. Indeed, it turns out that the min-parity condition over $\omega$ is the only infinitary Muller condition that guarantees positional determinacy on all game graphs.
\end{abstract}

\section{Motivation}

The problem of computing winning positions and winning strategies in infinite games has numerous applications in computing, most notably for the synthesis and verification of reactive controllers and for the model-checking of the $\mu$-calculus and other logics. Of special importance are parity games, due to several reasons.

2000 ACM Subject Classification: F.4.1, G2.

Key words and phrases: Games, logic, positional determinacy, parity games, Muller games.

* This research has been partially supported by the European Research Training Network "Games and Automata for Synthesis and Validation" (GAMES). 
(1) Many classes of games arising in practical applications admit reductions to parity games (over larger game graphs). This is the case for games modeling reactive systems, with winning conditions specified in some temporal logic or in monadic second-order logic over infinite paths (S1S), for Muller games, but also for games with partial information appearing in the synthesis of distributed controllers [1].

(2) Parity games arise as the model checking games for fixed point logics such as the modal $\mu$-calculus or LFP, the extension of first-order logic by least and greatest fixed points [11, 14]. In particular the model checking problem for the modal $\mu$-calculus can be solved in polynomial time if, and only if, winning regions for parity games can be computed in polynomial time.

(3) Parity games are positionally determined [10, 24]. This means that from every position, one of the two players has a winning strategy whose moves depend only on the current position, not on the history of the play. This property is fundamental for numerous results in automata theory on infinite objects and for verification algorithms.

In most of the traditional applications of games in computer science, the arena, and therefore also the number of priorities appearing in the winning condition, are finite. However, due to applications in the verification of infinite-state systems and other areas where infinite structures become increasingly important, it is interesting to study infinite arenas that admit some kind of finite presentation. The best studied class of such games are pushdown games [21, 28, where the arena is the configuration graph of a pushdown automaton. Other relevant classes of infinite, but finitely presented, (game) graphs include prefix-recognizable graphs, HR- and VR-equational graphs, graphs in the Caucal hierarchy, and automatic graphs. On all these classes of graphs (with the exception of automatic graphs [5]), monadic second-order logic can be evaluated effectively, which implies, for instance, that winning regions of parity games with a finite number of priorities are decidable. However, once we move to infinite game graphs, winning conditions depending on infinitely many priorities arise naturally. In pushdown games, stack height and stack contents are natural parameters that may take infinitely many values. In [7], Cachat, Duparc, and Thomas study pushdown games with an infinity condition on stack contents, and Bouquet, Serre, and Walukiewicz [6] consider more general winning conditions for pushdown games, combining a parity condition on the states of the underlying pushdown automaton with an unboudedness condition on stack heights. Similarly, Gimbert [12] considers games of bounded degree where the parity winning conditions is combined with the requirement that an infinite portion of the game graph is visited.

To establish positional determinacy or finite-memory determinacy is a fundamental first step in the analysis of an infinite game, and is also crucial for the algorithmic construction of winning strategies. In the case of parity games with finitely many priorities the positional determinacy immediately implies that winning regions can be decided in NP $\cap$ Co-NP; with a little more effort it follows that the problem is in fact in UP $\cap$ Co-UP [17]. Further, although it is not known yet whether parity games can be solved in polynomial time, all known approaches towards an efficient algorithmic solution make use of positional determinacy, including the presently best deterministic algorithm from [19]. The same is true for the polynomial-time algorithms that we have for specific classes of parity games, including parity games with a bounded number of priorities [18], games where even and odd cycles do not intersect, solitaire games and nested solitaire games [3], and parity games 
of bounded tree width [25], bounded entanglement [4], or bounded DAG-width [2, 26]. Positional determinacy is also the key point in the proofs of most of the known results on pushdown games.

In general, the positional determinacy of a game may depend on specific properties of the arena and on the winning condition. For instance, the previously known results on pushdown games make use of the fact that the arena is a pushdown graph. However, this is not always the case. As we show here, there are interesting cases, where positional determinacy is a consequence of the winning condition only. Most notably this is the case for the parity condition (little endian style) on $\omega$. In fact, we completely classify the infinitary Muller conditions with this property and show that they are equivalent to a parity condition. This result gives a general, arena-independent explanation of the positional determinacy of certain pushdown games. We hope and expect that it will be the first step for algorithmic solutions for other infinite games with finitely presented arenas.

\section{INTRODUCTION}

2.1. Games and strategies. We study two-player games of infinite duration on arenas with infinitely many priorities. An arena $\mathcal{G}=\left(V, V_{0}, V_{1}, E, \Omega\right)$, consists of a directed graph $(V, E)$, with a partioning $V=V_{0} \cup V_{1}$ of the nodes into positions of Player 0 and positions of Player 1. The possible moves are described by the edge relation $E \subseteq V \times V$. The function $\Omega: V \rightarrow C$ assigns to every position a priority. Occasionally we encode the priority function by the collection $\left(P_{c}\right)_{c \in C}$ of unary predicates where $P_{c}=\{v \in V: \Omega(v)=c\}$.

In case $(v, w) \in E$ we call $w$ a successor of $v$ and we denote the set of all successors of $v$ by $v E$. To avoid tedious case distinctions, we assume that every position has at least one successor. A play of $\mathcal{G}$ is an infinite path $v_{0} v_{1} \ldots$ formed by the two players starting from a given initial position $v_{0}$. Whenever the current position $v_{n}$ belongs to $V_{\sigma}$, then Player $\sigma$ chooses a successor $v_{n+1} \in v_{n} E$. A game is given by an arena and a winning condition that describes which of the plays $v_{0} v_{1} \ldots$ are won by Player 0 , in terms of the sequence $\Omega\left(v_{0}\right) \Omega\left(v_{1}\right) \ldots$ of priorities appearing in the play. Thus, a winning condition is a set $W \subseteq C^{\omega}$ of infinite sequences of priorities.

A (deterministic) strategy for Player $\sigma$ is a partial function $f: V^{*} V_{\sigma} \rightarrow V$ that assigns to finite paths through $\mathcal{G}$ ending in a position $v \in V_{\sigma}$ a successor $w \in v E$. A play $v_{0} v_{1} \cdots \in$ $V^{\omega}$ is consistent with $f$ if, for each initial segment $v_{0} \ldots v_{i}$ with $v_{i} \in V_{\sigma}$, we have that $v_{i+1}=f\left(v_{0} \ldots v_{i}\right)$. We say that such a strategy $f$ is winning from position $v_{0}$ if every play that starts at $v_{0}$ and that is consistent with $f$ is won by Player $\sigma$. The winning region of Player $\sigma$, denoted $W_{\sigma}$, is the set of positions from which Player $\sigma$ has a winning strategy. A game $\mathcal{G}$ is determined if $W_{0} \cup W_{1}=V$, i.e., if from each position one of the two players has a winning strategy.

Winning strategies can be rather complicated. Of special interest are simple strategies, in particular finite memory strategies and positional strategies. While positional strategies only depend on the current position, not on the history of the play, finite memory strategies have access to bounded amount of information on the past. Finite memory strategies can be defined as strategies that are realisable by finite automata.

More formally, a strategy with memory $M$ for Player $\sigma$ is given by a triple $\left(m_{0}, U, F\right)$ with initial memory state $m_{0} \in M$, a memory update function $U: M \times V \rightarrow M$ and a next-move function $F: V_{\sigma} \times M \rightarrow V$. Initially, the memory is in state $m_{0}$ and after the 
play has gone through the sequence $v_{0} v_{1} \ldots v_{m}$ the memory state is $u\left(v_{0} \ldots v_{m}\right)$, defined inductively by $u\left(v_{0} \ldots v_{m} v_{m+1}\right)=U\left(u\left(v_{0} \ldots v_{m}\right), v_{m+1}\right)$. In case $v_{m} \in V_{\sigma}$, the next move from $v_{1} \ldots v_{m}$, according to the strategy, leads to $F\left(v_{m}, u\left(v_{0} \ldots, v_{m}\right)\right)$. In case $M=\left\{m_{0}\right\}$, the strategy is positional; it can be described by a function $F: V_{\sigma} \rightarrow V$.

Definition 2.1. A game is positionally determined, if it is determined, and each player has a positional winning strategy on his winning region.

Clearly, if the arena is a forest, then all strategies are positional, so the game is positionally determined if, and only if, it is determined.

Throughout the paper, we assume the Axiom of Choice.

2.2. Games with infinitely many priorities. In the context of finite-memory determinacy or positional determinacy of infinite games it is usually assumed that the range of the priority function is finite, and the winning condition is defined by a formula on infinite paths (from S1S or LTL, say) referring to the predicates $\left(P_{c}\right)_{c \in C}$, or by an automata-theoretic condition like a Muller, Rabin, Streett, or parity (Mostowski) condition (see e.g. [15, 9, 29]). In Muller games the winner of a play depends only on the set of priorities that have been seen infinitely often; it has been proved by Gurevich and Harrington [16] that Muller games are determined and that the winner has a finite-memory winning strategy. An important special case of Muller games are parity games where the least (or greatest) priority occurring infinitely often determines the winner.

Here we will extend the study of positional determinacy to games with infinitely many priorities. Specifically we are interested in games with priority assignments $\Omega: V \rightarrow \omega$. Besides the obvious theoretical interest, such games arise in several areas. For instance, the winning conditions of pushdown games are specific instances of abstract winning conditions in games with infinitely many priorities. It is interesting to study these games in a general setting, and to isolate the winning conditions that lead to positional determinacy on arbitrary arenas, not just on specific ones like pushdown games.

Based on priority assigments $\Omega: V \rightarrow \omega$ we will first consider the following classes of games.

Infinity games: are games where Player 0 wins precisely those infinite plays in which no priority appears infinitely often.

Parity games: are games where Player 0 wins the infinite plays where the least priority seen infinitely often is even, or where all priorities appear only finitely often.

Max-parity games: are games where Player 0 wins if the maximal priority occurring infinitely often is even, or does not exist.

Note that we have chosen the definitions so that in case no priority appears infinitely often, the winner is always Player 0. It is clear that these games are determined, because the winning conditions are Borel sets, and a fundamental result due to Martin [22] states that all Borel games are determined. To be more precise, the infinity and parity winning conditions are on the $\boldsymbol{\Pi}_{3}^{0}$-level of the Borel hierarchy. Indeed, note that for any $m \in \omega$ the set $A_{m}$ of words that contain infinitely many occurences of $m$ is in $\boldsymbol{\Pi}_{2}^{0}$ since it is the countable intersection of the open sets $A_{m}^{n}:=\left(\omega^{*} m\right)^{n} \omega^{\omega}$, for all $n \in \omega$. Now the parity condition can be expressed as the the set of infinite words $x=x_{0} x_{1} x_{2} \ldots$ such that for all odd $m$, either $x \notin A_{m}$ or there is an even number $k<m$ such that $x \in A_{k}$. Similarly, it is easy to see that the max-parity condition is on the $\boldsymbol{\Delta}_{4}^{0}$-level of the Borel hierarchy. 
For games with only finitely many priorities, min-parity and max-parity winning conditions can be (and are) used interchangeably. This is not the case when we have infinitely many priorities.

Proposition 2.2. Max-parity games with infinitely many priorities in general do not admit finite memory winning strategies.

Proof. Consider the max-parity game with positions $V_{0}=\{0\}$ and $V_{1}=\{2 n+1: n \in \mathbb{N}\}$ (where the name of a position is also its priority), such that Player 0 can move from 0 to any position $2 n+1$ and Player 1 can move back from $2 n+1$ to 0 . Clearly Player 0 has a winning strategy from each position but no winning stategy with finite memory.

However, we will see that (min-)parity games with priorities in $\omega$ are positionally determined.

2.3. Strategy forests. Let $f$ be a strategy for Player $\sigma$ in the game $\mathcal{G}=\left(V, V_{0}, V_{1}, E, \Omega\right)$. For any initial position $v_{0}$ of the game, we can associate with $f$ the strategy tree $\mathcal{T}_{f}$, the tree of all plays that start at $v_{0}$ and that are consistent with $f$. In the obvious way, $\mathcal{I}_{f}$ can itself be considered as a game graph, with a canonical homomorphism $h: \mathcal{T}_{f} \rightarrow \mathcal{G}$. For every position $v$ of $\mathcal{G}$, we call the nodes $s \in h^{-1}(v)$ the occurrences of $v$ in $\mathcal{T}_{f}$. Since we assume that strategies are deterministic every occurence of a node $v \in V_{\sigma}$ has precisely one successor in the strategy forest $\mathcal{T}_{f}$, whereas every occurrence of a node $v \in V_{1}$ has precisely as many successors in $\mathcal{T}_{f}$ as $v$ has in $\mathcal{G}$. If $f$ is a winning strategy from $v_{0}$, then every path through $\mathcal{T}_{f}$ is a winning play for Player $\sigma$. If we consider a set of initial positions (like the entire winning region $W_{\sigma}$ ) then $\mathcal{T}_{f}$ is a strategy forest with a separate tree for each initial position.

By moving from game graphs to strategy forests we can eliminate the interaction between the two players and thus simplify the analysis. We already know that the games that we study are determined. To prove positional determinacy we proceed as follows.

We take a winning strategy and define a collection of well-founded pre-orders on its strategy forest. We then define a positional winning strategy for the original game, by copying for each position in the winning region, the winning stategy from a minimal occurrence of the position in the strategy tree. We then show that the resulting positional strategy is indeed winning.

To simplify the exposition we first discuss infinity games. Note that these can be seen as a special case of parity games. Indeed, if we change the priorities of an infinity game $\mathcal{G}$ so that all priorities become odd, by setting $\Omega^{\prime}(v):=2 \Omega(v)+1$, and replace the infinity winning condition by the parity condition, then the resulting parity game $\mathcal{G}^{\prime}$ is equivalent to $\mathcal{G}$.

\section{InFinity GAMES}

We start with some remarks on arbitrary transition systems. We will then apply them to strategy forests.

Given any transition system $\mathcal{K}=(S, E, P)$ with set of states $S$, transition relation $E$ and atomic proposition $P$, we assign to each state $s$ an ordinal $\alpha(s)$ or $\infty$. Informally, $\alpha(s)$ tells us how often a path from $s$ can hit $P$. To define this precisely, we proceed inductively. For any ordinal $\alpha$, let $X^{\alpha}$ be the set of all $s \in S$ such that whenever a path from $s$ hits a node 
$t \in P$, then all successors of $t$ belong to $\bigcup_{\beta<\alpha} X^{\beta}$. Finally, let $\alpha(s)=\min \left\{\alpha: s \in X^{\alpha}\right\}$. If $s$ is not contained in any $X^{\alpha}$, the we put $\alpha(s)=\infty$.

Remark. We can equivalently define $\alpha(s)$ in terms of closure ordinals in the modal $\mu$ calculus. Consider the formula $\mu X . \varphi(X)$, with $\varphi(X):=\nu Y .(P \rightarrow \square X) \wedge \square Y$. It expresses that on all paths, there are only finitely many occurrences of $P$. We define the stage $X^{\alpha}$ of the least fixed point induction via $\varphi(X)$ by $X^{\alpha}=\left\{s: \mathcal{K}, s \models \varphi\left(X^{<\alpha}\right)\right\}$ where $X^{<\alpha}:=\bigcup_{\beta<\alpha} X^{\beta}$. It is easily seen that this coincides with the definition given above.

Remark. Note that although $\mu X . \varphi(X)$ expresses that on every path there are only finitely many occurrences of $P$ the closure ordinals need not be finite. For a simple example, consider an infinite path $v_{0} v_{1} v_{2} \ldots$ without occurences of $P$ and attach to each $v_{n}$ another infinite path on which $P$ is seen precisely $n$ times. On all these attached paths, $\alpha(s)$ will take only finite values, but $\alpha\left(v_{n}\right)=\omega$ for all $n$.

The following lemma is a direct consequence of the definitions.

Lemma 3.1. Suppose that every path in $\mathcal{K}$ contains only finitely many occurrences of $P$ (i.e., $\mathcal{K}, s \models \mu X . \varphi(X)$ for all $s)$. Then $\alpha(s) \geq \alpha(t)$ for all edges $(s, t)$ of $\mathcal{K}$, and the inequality is strict for $s \in P$.

Assume next that we have a transition system $\mathcal{K}=\left(S, E, P_{0}, P_{1}, P_{2}, \ldots\right)$ with infinitely many atomic propositions $P_{n}$. Proceeding as above for $P_{n}$ instead of $P$, we obtain, for each $n$, a function $\alpha_{n}$ mapping states $s \in S$ to ordinals. The signature of $s$ is $\operatorname{sig}(s):=$ $\left\langle\alpha_{n}(s): n<\omega\right\rangle$; we compare signatures lexicographically. Further, for each $n<\omega$, let $\operatorname{sig}_{n}(s)=\left\langle\alpha_{0}(s), \ldots, \alpha_{n}(s)\right\rangle$ and let $s<_{n} t$ denote that $\operatorname{sig}_{n}(s)<\operatorname{sig}_{n}(t)$ (i.e., that the signature of $s$ is strictly smaller than the signature of $t$ on the first $n+1$ positions). Similarly, let $s \leq_{n} t$ denote that $\operatorname{sig}_{n}(s) \leq \operatorname{sig}_{n}(t)$.

Note that $s<_{n} t$ implies $s<_{n+1} t$ and that each pre-order $<_{n}$ is well-founded (i.e., all descending chains are finite). On the other side, when we have infinitely many $P_{n}$, the lexicographic order of unrestricted signatures admits infinite descending chains.

Theorem 3.2. Infinity games are positionally determined

Proof. Let $W_{0}$ and $W_{1}$ be the winning regions of the two players for the infinity game on the arena $\mathcal{G}$. Note that the situation for the two players is not symmetric, so we have to consider them separately.

Let $f$ be any winning strategy for Player 0 on $W_{0}$. If $f$ is positional then we are done. Otherwise, we consider the strategy forest $\mathcal{T}_{f}$ and the canonical homomorphism $h: \mathcal{T}_{f} \rightarrow \mathcal{G}$. In $\mathcal{T}_{f}$ every path is winning for Player 0 , and thus hits each $P_{n}$ only finitely often. Hence the functions $\alpha_{n}(s)$ are defined and satisfy the properties of Lemma 3.1 .

We define a positional strategy $f^{\prime}$ for Player 0 as follows. Select a function $s: W_{0} \rightarrow \mathcal{I}_{f}$ that associates with each vertex $v \in W_{0}$ of priority $n$ a $<_{n}$-minimal element $s(v) \in h^{-1}(v)$ (i.e., a $<_{n}$-minimal occurrence of $v$ in the strategy forest). If $v$, and hence also $s(v)$, is a node of Player 0 , then there is a unique successor $t$ of $s(v)$ in $\mathcal{T}_{f}$; define $f^{\prime}(v):=h(t)$. Further, we define values of $\alpha_{n}$ (and hence $\operatorname{sig}_{n}$ ) on $W_{0}$ by $\alpha_{n}(v):=\alpha_{n}(s(v))$.

We claim that $f^{\prime}$ is winning from each node $v_{0} \in W_{0}$. Otherwise there exists a play $v_{0} v_{1} v_{2} \ldots$ that is consistent with $f^{\prime}$ and winning for Player 1 . Let $n$ be the least priority seen infinitely often on this play; take a suffix of the play on which priorities smaller than $n$ do no longer occur. We claim that the values of $\operatorname{sig}_{n}$ never increase on this suffix.

To see this, consider a move from $v$ to $w$ in this suffix and the corresponding moves in $\mathcal{T}_{f}$ from $s:=s(v)$ to $t$ with $h(t)=w$. By construction, and since $v$ and $w$ have priorities $\geq n$, 
we have $\operatorname{sig}_{n}(v)=\operatorname{sig}_{n}(s)$ and $\operatorname{sig}_{n}(w) \leq \operatorname{sig}_{n}(t)$. By Lemma 3.1, we have $\alpha_{m}(s) \geq \alpha_{m}(t)$ for all $m$ and the inequality is strict if $m$ is the priority of $s$. It follows that

$$
\operatorname{sig}_{n}(v)=\operatorname{sig}_{n}(s) \geq \operatorname{sig}_{n}(t) \geq \operatorname{sig}_{n}(w)
$$

and $\operatorname{sig}_{n}(v)>\operatorname{sig}_{n}(w)$ in case $v \in P_{n}$. Since there are infinitely many nodes $v_{i_{1}}, v_{i_{2}}, \ldots$ of priority $n$ in the suffix, we obtain an infinite descending chain

$$
\operatorname{sig}_{n}\left(v_{i_{1}}\right)>\operatorname{sig}_{n}\left(v_{i_{2}}\right)>\cdots
$$

which is impossible. Hence $f^{\prime}$ is indeed a winning strategy.

We now consider the case of Player 1 . Let $g$ be a strategy for Player 1 on $W_{1}$, with strategy forest $\mathcal{T}_{g}$ and canonical homomorphism $h: \mathcal{T}_{g} \rightarrow \mathcal{G}$. We define the 0-ancestor of a node $s \in \mathcal{T}_{g}$ to be the closest ancestor of $s$ that has priority 0 . Note that 0 -ancestors may be undefined. More generally, the $m$-ancestor of $s$ is the closest ancestor of priority $m$, provided it lies between $s$ and the $j$-ancestor of $s$, for all $j<m$ for which the $j$ ancestor is defined. We can thus associate with every node $s$ of priority $m$ an $(m+1)$-tuple $a(s)=\left\langle a_{0}(s), \ldots, a_{m}(s)\right\rangle \in\left(\mathcal{T}_{g} \cup\{\perp\}\right)^{m+1}$ of ancestors, where $a_{i}(s)=\perp$ means that the $i$-th ancestor of $s$ is not defined. Observe that $a_{m}(s)=s$ as $s$ is an ancestor of itself.

We fix a well-order $<$ on $\mathcal{T}_{g} \cup\{\perp\}$ (with maximal element $\perp$ ) and we compare tuples of ancestors via the lexicographical order that is induced by $<$. We can then associate with every $v \in W_{1}$ of priority $m$ the node $s(v) \in h^{-1}(v)$ with the minimal tuple of ancestors. Note that $s(v)$ is well-defined, because every position $v \in W_{1}$ has at least one occurrence $s \in \mathcal{T}_{g}$, and $a_{m}(s)=s$ if $m$ has priority $m$ (so at least one ancestor is defined). We extend the ancestor function to $W_{1}$ by setting $a(v):=a(s(v))$; this assigns to every node in $W_{1}$ a tuple of ancestors in $\mathcal{T}_{g}$. To define the positional strategy $g^{\prime}$, we select for any $v \in V_{1} \cap W_{1}$ the unique successor $t$ of $s(v)$ and set $g^{\prime}(v):=h(t)$.

We claim that this strategy is winning for Player 1. Suppose conversely that there is a losing play respecting the strategy. Then no priority appears infinitely often on this play. Consider the suffix of the play after the last appearance of priority 0 . Let us look at the 0 -ancestors of the positions in this suffix. These ancestors can only get smaller as the play proceeds. Indeed a move from $v$ to $w$ in such a play corresponds to a move from $s(v)$ to $t$ in $\mathcal{T}_{g}$ with $h(t)=w$. Since $w$ does not have priority $0, a_{0}(s)=a_{0}(t)$ and therefore $a_{0}(v)=a_{0}(s)=a_{0}(t) \geq a_{0}(w)$. This means that from some moment on all positions in the play will have the same 0 -ancestor. Consider the suffix of the play consisting only of these vertices. Next do the same with priority 1 . We find a position after which the 1-ancestor stabilises. Observe that it is a descendant of the 0 -ancestor and that there is no occurrence of priority 0 on the path between the two. Proceeding in this way we construct a path in the strategy tree $\mathcal{T}_{g}$ on which no priority appears infinitely often. But this is impossible, since $g$ was a winning strategy for Player 1 .

\section{PARity GAmes}

For parity games we proceed quite similarly, but we have to consider more complicated orderings on the strategy forests.

Consider a transition system $\mathcal{K}=(S, E, P, Q)$ with two atomic propositions $P$ and $Q$. We assign to each state $s$ an ordinal $\beta(s)$ or $\infty$ which, informally, tells us how often a path from $s$ can hit $P$ before seeing $Q$. Let $X^{0}$ be the set of all $s$ such that all paths from $s$ hit $Q$ before hitting $P$, and for $\beta>0$, let $X^{\beta}$ be the set of all $s$ such that whenever 
a path from $s$ hits a node $t \in P$, then all successors of $t$ belong to $X^{<\beta}$. Finally, let $\beta(s)=\min \left\{\beta: s \in X^{\beta}\right\}$.

Again, we have an equivalent definition in terms of the modal $\mu$-calculus. This time, consider the formula $\mu X . \varphi(X)$, with $\varphi(X):=\nu Y .(\neg P \vee \square X) \wedge(Q \vee \square Y)$. It expresses that on all paths, there are only finitely many occurrences of $P$ before seeing $Q$. Then $\beta(s)$ is the stage at which the least fixed point induction defined by $\varphi(X)$ becomes true at node $s$.

Lemma 4.1. Suppose that every path in $\mathcal{K}$ contains only finitely many occurrences of $P$ before hitting $Q$. Then $\beta(s) \geq \beta(t)$ for all edges $(s, t)$ of $\mathcal{K}$ with $s \notin Q$, and the inequality is strict for $s \in P$.

For infinity games we have defined ordinals $\alpha_{n}(s)$ telling us how often a path from $s$ can see priority $n$, independently for each $n$. Now we need different bounds $\beta_{n}$ which, informally, describe how often a path can hit the odd priority $n$ before seeing a smaller one.

Let $\mathcal{G}$ be a parity game, and let $\mathcal{T}_{f}=\left(S, E, P_{0}, P_{1}, P_{2}, \ldots\right)$ be the strategy forest of a winning strategy $f$ for Player 0 . Note that for every odd priority $n$, each path through $\mathcal{T}_{f}$ sees only finitely many occurrences of $n$ before seeing a priority $<n$. Hence, proceeding as above for $P:=P_{n}$ and $Q:=\bigcup_{m<n} P_{m}$ we obtain, for each odd $n$, a function $\beta_{n}$ mapping nodes $s \in \mathcal{T}_{f}$ to ordinals. The 0 -signatures of $s$ are $\operatorname{sig}_{n}^{0}(s):=\left\langle\beta_{1}(s), \beta_{3}(s) \ldots, \beta_{n^{\prime}}(s)\right\rangle$, where $n^{\prime}=n$ for odd $n$ and $n^{\prime}=n-1$ for even $n$; let $s<_{n}^{0} t$ denote that $\operatorname{sig}_{n}^{0}(s)<\operatorname{sig}_{n}^{0}(t)$. Further, $s \leq_{n}^{0} t$ means that $\operatorname{sig}_{n}^{0}(s) \leq \operatorname{sig}_{n}^{0}(t)$.

For strategy forests $\mathcal{T}_{g}$ of winning strategies of Player 1 , we proceed dually, associating with every node $s$ ordinals $\beta_{n}(s)$, for even $n$. We then define 1-signatures $\operatorname{sig}_{n}^{1}(s)=$ $\left\langle\beta_{0}(s), \beta_{2}(s), \ldots, \beta_{n^{\prime}}(s)\right\rangle$ (where $n^{\prime}$ is the largest even number not exceeding $n$ ) and the corresponding signature orderings $<_{n}^{1}$.

Again, we immediatley see that $s<_{n}^{i} t$ implies $s<_{n+1}^{i} t$ and that each $\leq_{n}^{i}$ is a wellfounded. Further, these orderings have very useful properties on strategy forests.

Lemma 4.2. Let $\mathcal{T}_{f}$ be the strategy forest associated with a winning strategy for Player 0 for a parity game. Then $t \leq_{\Omega(s)}^{0} s$ for all edges $(s, t)$ of $\mathcal{T}_{f}$ and the inequality is strict if $\Omega(s)$ is odd. In a strategy forest $\mathcal{T}_{g}$ of Player 1 , we have $t \leq_{\Omega(s)}^{1}$ s for all edges $(s, t)$ and the inequality is strict if $\Omega(s)$ is even.

Proof. If $(s, t)$ is an edge in $\mathcal{T}_{f}$, then by Lemma 4.1, $\beta_{m}(t) \leq \beta_{m}(s)$ for $m \leq \Omega(s)$, and, if $n=\Omega(s)$ is odd, and $\beta_{n}(t)<\beta_{n}(s)$. Similarly for $\mathcal{T}_{g}$.

Theorem 4.3. Parity games with priorities in $\omega$ are positionally determined.

Proof. The proof for Player 0 is precisely the same as for infinity games, using 0 -signatures and the associated orderings $<_{n}^{0}$.

For Player 1 we combine the approach for infinity games based on ancestors in the strategy forest with comparisons based on 1-signatures. As in the proof of Theorem 3.2 we associate with every node $s$ of priority $m$ in the strategy tree $\mathcal{T}_{g}$ the $(m+1)$-tuple $a(s)=\left\langle a_{0}(s), \ldots, a_{m}(s)\right\rangle \in\left(\mathcal{T}_{g} \cup\{\perp\}\right)^{m+1}$ of ancestors.

For each $i \in \omega$ we fix a well-order $\triangleleft_{i}$ extending $<_{i}^{1}$. Moreover we assume that $\perp$ is bigger in the $\triangleleft_{i}$-order than all the nodes. Let $s, s^{\prime}$ be two nodes of $\mathcal{T}_{g}$ of the same priority $m$. We write $s \prec_{m} s^{\prime}$ if there is $i \leq m$ such that $a_{i}(s) \triangleleft_{i} a_{i}\left(s^{\prime}\right)$ and $a_{j}(s)=a_{j}\left(s^{\prime}\right)$ for all $j<i$. Observe that $\prec_{m}$ is a well order on vertices of priority $m$.

For any position $v \in W_{1}$ of priority $m$ we now take the $\prec_{m}$-minimal occurrence $s(v)$ in $\mathcal{T}_{g}$ and define ancestors by $a(v):=a(s(v))$. For $v \in V_{1} \cap W_{1}$ we consider the unique 
successor $t$ of $s(v)$ and set $g^{\prime}(v):=h(t)$. This defines a positional strategy $g^{\prime}$ for Player 1 on $\mathcal{G}$.

We claim that this strategy is winning on $W_{1}$. Suppose conversely that there is a losing play respecting the strategy. Then either no priority appears infinitely often on this play, or the smallest priority occurring infinitely often is even.

If no priority occurs infinitely often, then we can proceed as in the proof of Theorem 3.2 to show that all ancestors eventually stabilise on the play, and thus obtain an infinite path in $\mathcal{T}_{g}$ on which no priority appears infinitely often. This is impossible since $g$ is a winning strategy for Player 1 . If the minimal priority $p$ appearing infinitely often is even, then we consider a suffix of the play that contains only priorities $\geq p$. By the same reasoning as in the first case it follows that all $q$-ancestors, for $q<p$, eventually stabilise on the play. Consider the suffix of the play after this has happened. A move from $v$ to $w$ on this suffix corresponds to a move from $s(v)$ to $t$ in $\mathcal{T}_{g}$. By definition, the $p$-ancestor of $v$ is $a_{p}(v)=a_{p}(s(v))$ and $a_{p}(w) \preceq_{p} a_{p}(t)$. On $\mathcal{T}_{g}$ we obviously have $a_{p}(t)=t$ if $\Omega(t)=p$ and $a_{p}(t)=a_{p}(s(v))$ if $\Omega(t)>p$. Now $t$ is a descendant of $a_{p}(s)$, so by Lemma 4.2 we have $t<_{p}^{1} a_{p}(s)$; for the case that $\Omega(t)=p$ this means that $a_{p}(t)=t<_{p}^{1} a_{p}(s)$. Since $\prec_{p}$ extends $<_{p}^{1}$ on nodes of priority $p$, we have that $a_{p}(w) \preceq_{p} a_{p}(v)$ and that the inequality is strict if $\Omega(w)=p$. But on the suffix we have an inifinite sequence of positions with priority $p$, and hence an infinite $\prec_{p}$-decreasing chain of $p$-ancestors, which is impossible.

Remark: Parity games over larger ordinals. We can also define parity games with a priority function $\Omega: V \rightarrow \alpha$ taking values in a larger set of ordinals than $\omega$. Recall that any ordinal can be written in a unique way as a sum $\lambda+n$ where $\lambda$ is a limit ordinal and $n<\omega$. We call $\lambda+n$ even if $n$ is. The question arises whether the positional determinacy of parity games over $\omega$ extends to larger ordinals. However, a tiny modification of the game in Proposition 2.2 shows that this is not the case. Indeed, if we replace in that game priority 0 by $\omega$, and use the (min-)parity winning condition, then Player 0 has a winning strategy from each position but no winning strategy with finite memory. For larger ordinals, a similar construction applies. This proves that parity games over ordinals $\alpha>\omega$ in general do not guarantee finite memory winning strategies.

Essentially the same construction shows that finite-memory determinacy also fails for some other variants of parity games over $\omega$, such as

- parity games where the priority function is partial (i.e., not all vertices have a priority),

- parity games with priorities on edges rather than vertices.

\section{Muller games}

Why do parity games and max parity games behave differently? Both are Muller conditions (i.e. they refer only to the set of priorities seen infinitely often) and the question arises which properties of Muller conditions are responsible for positional determinacy or determinacy with finite memory. In this section we assume that the set of priorities is countable. This is reasonable as on each play one can see only a countable number of them.

Definition 5.1. A Muller condition over a set $C$ of priorities is written in the form $\left(\mathcal{F}_{0}, \mathcal{F}_{1}\right)$ where $\mathcal{F}_{0} \subseteq \mathcal{P}(C)$ and $\mathcal{F}_{1}=\mathcal{P}(C)-\mathcal{F}_{0}$. A play in a game with Muller winning condition $\left(\mathcal{F}_{0}, \mathcal{F}_{1}\right)$ is won by Player $\sigma$ if, and only if, the set of priorities seen infinitely often in the play belongs to $\mathcal{F}_{\sigma}$. 
For infinity games, we have $\mathcal{F}_{0}=\{\emptyset\}$ and $\mathcal{F}_{1}=\mathcal{P}(\omega)-\{\emptyset\}$. For parity games,

$$
\begin{aligned}
& \mathcal{F}_{0}=\{X \subseteq \omega: \min (X) \text { is even }\} \cup\{\emptyset\} \\
& \mathcal{F}_{1}=\{X \subseteq \omega: \min (X) \text { is odd }\}
\end{aligned}
$$

For max-parity games, we have

$$
\begin{aligned}
\mathcal{F}_{0}=\{ & X \subseteq \omega: \text { if } X \text { is finite and non-empty, } \\
& \text { then } \max (X) \text { is even }\} \\
\mathcal{F}_{1}=\{ & X \subseteq \omega: X \text { is finite, non-empty, and } \\
& \max (X) \text { is odd }\}
\end{aligned}
$$

Definition 5.2. We say that $\left(\mathcal{F}_{0}, \mathcal{F}_{1}\right)$ guarantees positional winning strategies if all games with winning condition $\left(\mathcal{F}_{0}, \mathcal{F}_{1}\right)$ are positionally determined.

Following McNaughton [23] and Zielonka [29] we say that $\mathcal{F}_{\sigma}$ has a strong split if there exist sets $X_{0}, X_{1} \in \mathcal{F}_{\sigma}$ with $X_{0} \cap X_{1} \neq \emptyset$ and $X_{0} \cup X_{1} \in \mathcal{F}_{1-\sigma}$. Zielonka [29] has shown that a Muller condition over a finite set of priorities guarantees positional winning strategies if, and only if,

(P0): $\mathcal{F}_{0}$ and $\mathcal{F}_{1}$ have no strong splits.

Remark. A weak split is a pair of disjoint sets with $X_{0}, X_{1} \in \mathcal{F}_{\sigma}$ and $X_{0} \cup X_{1} \in \mathcal{F}_{1-\sigma}$. Muller conditions over finite sets of priorities may have weak splits and still guarantee positional winning strategies. The simplest case is when $\mathcal{F}_{0}$ consists of the set $\{0,1\}$, but $\{0\}$ and $\{1\}$ belong to $\mathcal{F}_{1}$.

We want to find a similar characterisation of Muller conditions with positional winning strategies for the case of infinite sets of priorities.

We observe that for infinity games and parity games $\mathcal{F}_{0}$ and $\mathcal{F}_{1}$ are closed under unions and non-empty intersections of chains:

(P1): For every infinite descending chain $X_{1} \supseteq X_{2} \supseteq \ldots$ of elements of $\mathcal{F}_{\sigma}$ either $\bigcap_{i<\omega} X_{i}=\emptyset$ or it is an element of $\mathcal{F}_{\sigma}$.

(P2): For every chain $X_{1} \subseteq X_{2} \subseteq \ldots$ of elements of $\mathcal{F}_{\sigma}$, also $\bigcup_{i<\omega} X_{i}$ belongs to $\mathcal{F}_{\sigma}$.

On the other side, for the max-parity condition, $\mathcal{F}_{0}$ is not closed under non-empty intersections of chains (take $X_{i}=\{1\} \cup\{n: n>i\}$ ) and $\mathcal{F}_{1}$ is not closed under unions of chains (take $X_{i}=\{j: j \leq 2 i+1\}$ ). Condition (P1) fails also for min-parity condition for ordinals $\alpha>\omega$. Indeed we have $\mathcal{F}_{1}=\{X \subseteq \alpha: \min (X)$ is odd $\}$ which is not closed under non-empty intersections of chains (take $X_{i}=\{\omega\} \cup\{n: 2 i+1 \leq n<\omega\}$ ).

We will show first, that condition (P1) is necessary for the positional determinacy of a Muller condition.

Lemma 5.3. If there is an infinite sequence $X_{1} \supseteq X_{2} \supseteq \cdots$ of elements of $\mathcal{F}_{1-\sigma}$ with $\bigcap X_{i}=Y \neq \emptyset$ and $Y \in \mathcal{F}_{\sigma}$ then there is game with winning condition $\left(\mathcal{F}_{0}, \mathcal{F}_{1}\right)$ that Player $\sigma$ wins, but needs infinite memory to do so.

Proof. Consider the following game where circles denote positions of Player $\sigma$ and boxes positions of Player $(1-\sigma)$. 


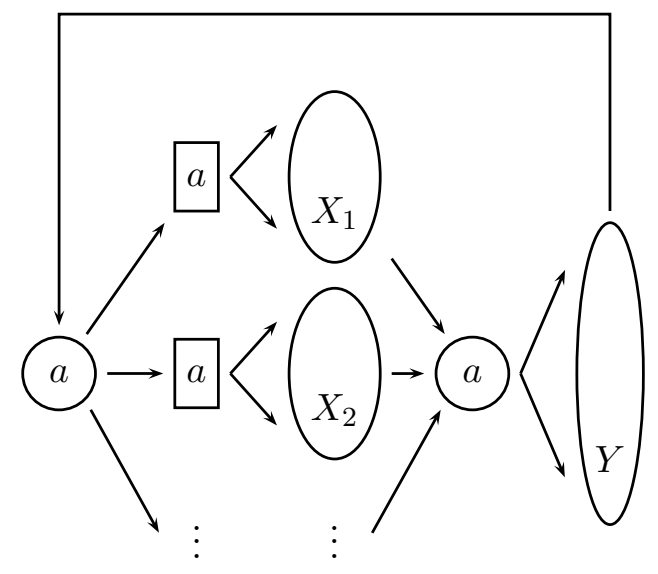

Here $a$ is some arbitrary element of $Y$. A play in this game is an infinite sequence of subplays; in each subplay Player $\sigma$ first decides from which $X_{i}$ the opponent is going to choose next. After Player $(1-\sigma)$ has made his choice, Player $\sigma$ can select an element from $Y$.

If Player $\sigma$ allows her opponent to choose from some $X_{i}$ infinitely often then Player (1$\sigma)$ can make all elements of $X_{i}$ appear infinitely often on the play. This means that in order not to lose, Player $\sigma$ must permit Player $(1-\sigma)$ to choose from each $X_{i}$ only finitely often. If she does this then she wins as she can make sure that each element of $Y$ is seen infinitely often thanks to the last part of the each subplay. Thus Player $\sigma$ has a winning strategy, but none that uses only finite memory.

To show the necessity of conditions (P0) and (P2) we consider the following game.

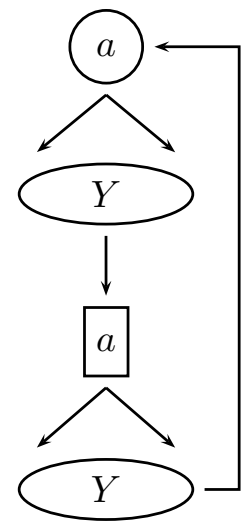

Here, $Y$ is a set and $a \in Y$. The arrows to the ovals with $Y$ mean that the player can choose any element of $Y$. Clearly, if $Y \in \mathcal{F}_{\sigma}$, then Player $\sigma$ can win by visiting all elements of $Y$ infinitely often. However, if Player $\sigma$ plays memoryless then she must select a fixed element $b$, and her opponent can chose an arbitrary set $X \subseteq Y$ of nodes and make sure that the set of nodes visited infinitely often is $\{a, b\} \cup X$. More generally, if Player $\sigma$ plays with a finite memory strategy this amounts to selecting a finite set $B$; Player $(1-\sigma)$ can then win if there exists a set $X \in \mathcal{F}_{1-\sigma}$ with $\{a\} \cup B \subseteq X \subseteq Y$.

Lemma 5.4. If $\mathcal{F}_{1-\sigma}$ contains a strong split, then there is a game with winning condition $\left(\mathcal{F}_{0}, \mathcal{F}_{1}\right)$ that is won by Player $\sigma$, but not with a positional strategy. 
Proof. Let $X_{0} \cup X_{1} \in \mathcal{F}_{\sigma}$ with $X_{0}, X_{1} \in \mathcal{F}_{1-\sigma}$ and $X_{0} \cap X_{1} \neq \emptyset$. Take the game above with $Y=X_{0} \cup X_{1}$ and $a \in X_{0} \cap X_{1}$. Player $\sigma$ wins since $Y=X_{0} \cup X_{1} \in \mathcal{F}_{\sigma}$. However, she cannot win positionally. Indeed the single element $b$ selected by a positional strategy of Player $\sigma$ belongs to $X_{i}(i=0$ or 1$)$, and Player $1-\sigma$ can win by making sure that all elements of $X_{i}$, and only these, are visited infinitely often.

Lemma 5.5. If $\mathcal{F}_{1-\sigma}$ is not closed under unions of chains, then there is a game with winning condition $\left(\mathcal{F}_{0}, \mathcal{F}_{1}\right)$ that Player $\sigma$ wins, but needs infinite memory to do so.

Proof. Let $X_{1} \subseteq X_{2} \subseteq \cdots$ be an infinite ascending chain in $\mathcal{F}_{1-\sigma}$ with $\bigcup_{i} X_{i} \in \mathcal{F}_{\sigma}$. Take the game described above with $Y=\bigcup_{i} X_{i}$ and $a \in X_{1}$. Again Player $\sigma$ wins since $Y \in \mathcal{F}_{\sigma}$. But if Player $\sigma$ plays with finite memory, this amounts to selecting a finite set $B \subseteq Y$ of elements that she visits infinitely often. Since $B$ is finite $B \subseteq X_{i}$ for some $i$; hence Player $1-\sigma$ can make sure that the set of elements visited infinitely often is $X_{i}$ and wins.

In the remaining part of the section we will characterise the Muller conditions satisfying $(\mathrm{P} 0),(\mathrm{P} 1)$ and (P2) in a different way, via Zielonka paths, and then show that any such condition can be reformulated as a parity condition over an ordinal $\alpha \leq \omega$. In particular, this implies that these closure properties are necessary and sufficient to guarantee positional determinacy on all game graphs.

Definition 5.6. The Zielonka tree of a Muller condition $\left(\mathcal{F}_{0}, \mathcal{F}_{1}\right)$ over $C$ is a tree $Z\left(\mathcal{F}_{0}, \mathcal{F}_{1}\right)$ whose nodes are labelled with pairs $(X, \sigma)$ such that $X \in \mathcal{F}_{\sigma}$. Let $\sigma$ be the player that wins with the set of all priorities, i.e. $C \in \mathcal{F}_{\sigma}$ with $C=\bigcup \mathcal{F}_{0} \cup \bigcup \mathcal{F}_{1}$. The Zielonka tree $Z\left(\mathcal{F}_{0}, \mathcal{F}_{1}\right)$ exists, if for every maximal $Y \in \mathcal{F}_{1-\sigma}$ the Zielonka tree $Z\left(\mathcal{F}_{0} \cap \mathcal{P}(Y), \mathcal{F}_{1} \cap \mathcal{P}(Y)\right)$ exists and every set in $\mathcal{F}_{1-\sigma}$ is a subset of some maximal set in $\mathcal{F}_{1-\sigma}$. In that case $Z\left(\mathcal{F}_{0}, \mathcal{F}_{1}\right)$ consists of a root, labeled by $(C, \sigma)$, to which we attach as subtrees the Zielonka trees $Z\left(\mathcal{F}_{0} \cap \mathcal{P}(Y), \mathcal{F}_{1} \cap \mathcal{P}(Y)\right.$ ), for the maximal sets $Y \in \mathcal{F}_{1-\sigma}$. (In particular, if $\mathcal{F}_{1-\sigma}=\emptyset$, then the Zielonka tree consists of a single node.)

For Muller conditions over a finite set $C$, the Zielonka tree always exists, and it is a fundamental tool for analysing the memory that is required for solving Muller games [9]. For infinite sets $C$, the Zielonka tree need not exist, since there is no guarantee, that for $X \in \mathcal{F}_{\sigma}$, the set $\mathcal{P}(X) \cup \mathcal{F}_{1-\sigma}$ contains maximal elements. For instance the max-parity condition does not have a Zielonka tree.

Proposition 5.7. For every Muller condition satisfying property (P2) the Zielonka tree exists.

Proof. By (P2) the union over any chain $Y_{0} \subseteq Y_{1} \subseteq \ldots$ in $\mathcal{P}(X) \cap \mathcal{F}_{\sigma}$ is again contained $\mathcal{P}(X) \cap \mathcal{F}_{\sigma}$. Hence, by Zorn's Lemma, $\mathcal{P}(X) \cap \mathcal{F}_{\sigma}$ has maximal elements.

Now let $S$ be the set of elements of $\mathcal{P}(X) \cap \mathcal{F}_{\sigma}$ that are not below a maximal element. For any $Y \in S$ there exists a set $Y^{\prime} \supsetneq Y$ which must again belong to $S$. Further, the union over any chain in $S$ is again contained in $S$. If $S$ were non-empty, then, again by Zorn's Lemma, $S$ would contain maximal elements, which is absurd.

We say that a Muller condition $\left(\mathcal{F}_{0}, \mathcal{F}_{1}\right)$ is described by a Zielonka path of co-finite sets if the Zielonka tree $Z\left(\mathcal{F}_{0}, \mathcal{F}_{1}\right)$ exists, and it is a finite or infinite path, consisting of co-finite sets, and possibly the empty set at the end.

Proposition 5.8. Every Muller condition on a countable set $C$, satisfying properties (P0), (P1), and (P2), is described by a Zielonka path of co-finite sets. 
Proof. We already know that the Zielonka tree for $\left(\mathcal{F}_{0}, \mathcal{F}_{1}\right)$ exists. The set that labels the root of the Zielonka tree is $C$ which is co-finite. Consider now any node of the Zielonka tree, labelled $(X, 1-\sigma)$. If all subsets of $X$ belong to $\mathcal{F}_{1-\sigma}$ (in particular if $X=\emptyset$ ), then the node is a leaf of the Zielonka tree. Otherwise, by Proposition 5.7, we know that $\mathcal{P}(X) \cap \mathcal{F}_{\sigma}$ contains a maximal element $Y$. If $X \backslash Y$ was infinite then one could consider any infinite descending chain $X_{1} \supsetneq X_{2} \supsetneq \ldots$ of sets in $\mathcal{P}(X) \cap \mathcal{F}_{1-\sigma}$ whose intersection is $Y$. But, unless $Y=\emptyset$, this would violate property (P1). Hence, $Y$ is either co-finite or empty. Now suppose that there are two distinct maximal elements $Y_{1}, Y_{2}$ in $\mathcal{P}(X) \cap \mathcal{F}_{\sigma}$. Since $Y_{1}, Y_{2}$ are both co-finite, $Y_{1} \cap Y_{2} \neq \emptyset$. By property (P0), $Y_{1} \cup Y_{2} \in \mathcal{F}_{\sigma}$ which is impossible by the maximality of $Y_{1}$ and $Y_{2}$. This means that the node $(X, 1-\sigma)$ has a unique sucessor $(Y, \sigma)$, with $Y$ being the greatest element in $\mathcal{P}(X) \cap \mathcal{F}_{\sigma}$.

Thus, the Zielonka tree is indeed a finite or infinite path of co-finite sets and, if it is finite, with possibly the empty set at the end.

Next we have to make precise what it means that a Muller condition reduces to a parity condition.

Definition 5.9. A Muller condition $\left(\mathcal{F}_{0}, \mathcal{F}_{1}\right)$ on $C$, with $\emptyset \in \mathcal{F}_{\sigma}$, reduces to a parity condition on $\alpha$, if there is a function $f: C \rightarrow \alpha$ such that,

(1) for every non-empty $X \subseteq C$ we have that

$$
X \in \mathcal{F}_{\sigma} \Longleftrightarrow \min f(X) \text { is even, }
$$

(2) $f^{-1}(d)$ is finite for every $d \in \alpha$, unless $d=\max f(C)$ and $d$ is even.

Note that such a reduction may have to switch the role of the two players. Indeed, if $\emptyset \in \mathcal{F}_{1}$, then the role of Player 1 in the Muller game must be taken by Player 0 in the parity game since, by convention, a play of a parity game in which no priority is seen infinitely often is won by Player 0 .

Proposition 5.10. If a Muller condition $\left(\mathcal{F}_{0}, \mathcal{F}_{1}\right)$ reduces to a parity game on some $\alpha \leq \omega$, then it guarantees positional winning strategies.

Proof. Since $\left(\mathcal{F}_{0}, \mathcal{F}_{1}\right)$ guarantees positional winning strategies if, and only if, $\left(\mathcal{F}_{1}, \mathcal{F}_{0}\right)$ does, we may assume that $\emptyset \in \mathcal{F}_{0}$. With the function $f: C \rightarrow \alpha$ we can relabel any Muller game $\mathcal{G}$ with winning condition $\left(\mathcal{F}_{0}, \mathcal{F}_{1}\right)$ to a parity game $\mathcal{G}^{\prime}$ on the same game graph. Since $\mathcal{G}^{\prime}$ is positionally determined it suffices to show that every play $\pi$ in $\mathcal{G}$ is won by the same player as the corresponding play in $\mathcal{G}^{\prime}$. Let $X$ be the set of priorities occurring infinitely often in $\pi$. In the corrsponding play in $\mathcal{G}^{\prime}$, the set of priorities seen infinitely often is either $f(X)$ or $f(X) \cup\{d\}$, where $d=\max f(C)$ is even. If $X$ is empty, then so is $f(X)$, and hence the minimal priority seen infinitely often in $\pi^{\prime}$ is either $d$, which is even, or does not exist. Otherwise, $X \in \mathcal{F}_{0}$ if, and only if, $\min f(X)$ is even. Hence Player 0 wins $\pi$ if, and only if, she wins $\pi^{\prime}$.

We want to prove that any Muller condition described by a Zielonka path of co-finite sets reduces to a parity condition. Before we do so, we illustrate the reduction by two examples. First, consider the case that, for certain $a, b, c, d \in \omega$,

$$
\begin{aligned}
& \mathcal{F}_{0}=\{X \subseteq \omega: a \in X \vee b \in X \vee\{c, d\} \cap X=\emptyset\} \\
& \mathcal{F}_{1}=\{X \subseteq \omega:\{a, b\} \cap X=\emptyset \wedge(c \in X \vee d \in X)\}
\end{aligned}
$$


The Zielonka path for $\left(\mathcal{F}_{0}, \mathcal{F}_{1}\right)$ is

$$
(\omega, 0) \longrightarrow(\omega \backslash\{a, b\}, 1) \longrightarrow(\omega \backslash\{a, b, c, d\}, 0)
$$

and we can immediately read off an appropriate reduction $f$ from $\left(\mathcal{F}_{0}, \mathcal{F}_{1}\right)$ to a parity condition with three priorities, namely $f(a)=f(b)=0, f(c)=f(d)=1$, and $f(x)=2$ for all other $x \in \omega$. However, if we change the condition just a little bit, by moving the empty set from $\mathcal{F}_{0}$ to $\mathcal{F}_{1}$, the reduction becomes quite different. The Zielonka path now has the form

$$
(\omega, 0) \longrightarrow(\omega \backslash\{a, b\}, 1) \longrightarrow(\omega \backslash\{a, b, c, d\}, 0) \longrightarrow(\emptyset, 1) .
$$

Since $\emptyset \in \mathcal{F}_{1}$ we have to change the role of the players. Moreover, we can no longer map all elements of $\omega \backslash\{a, b, c, d\}$ to the same priority since a play in the Muller game may see this set infinitely often without seeing any of of its elements more than a finite number of times. Hence an appropriate reduction $f: \omega \rightarrow \omega$ is now defined by

$$
f(x)= \begin{cases}1 & \text { for } x=a \text { and } x=b \\ 2 & \text { for } x=c \text { and } x=d \\ 2 x+3 & \text { for } x \in \omega \backslash\{a, b, c, d\} .\end{cases}
$$

Proposition 5.11. Every Muller condition that is described by a Zielonka path of co-finite sets reduces to a parity game on an ordinal $\alpha \leq \omega$.

Proof. Let $\left(\mathcal{F}_{0}, \mathcal{F}_{1}\right)$ be a Muller condition with $\emptyset \in \mathcal{F}_{0}$. Otherwise we replace $\left(\mathcal{F}_{0}, \mathcal{F}_{1}\right)$ by $\left(\mathcal{F}_{1}, \mathcal{F}_{0}\right)$.

The Zielonka path for $\left(\mathcal{F}_{0}, \mathcal{F}_{1}\right)$ gives, for some $\beta \leq \omega$ a descending sequence $\left(Z_{i}\right)_{0 \leq i<\beta}$ (in case $C \in \mathcal{F}_{0}$ ) or $\left(Z_{i}\right)_{1 \leq i<\beta}$ (in case $C \in \mathcal{F}_{1}$ ), which consists of co-finite sets, and possibly the empty set at the end, such that

- $Z_{2 i} \in \mathcal{F}_{0}, Z_{2 i+1} \in \mathcal{F}_{1}$.

- If $Y \subseteq Z_{2 i}$ and $Y \nsubseteq Z_{2 i+1}$, then $Y \in \mathcal{F}_{0}$. Similarly, if $Y \subseteq Z_{2 i+1}$ and $Y \nsubseteq Z_{2 i+2}$, then $Y \in \mathcal{F}_{1}$.

To define the reduction $f: C \rightarrow \alpha$, we distinguish three cases.

(1) If the Zielonka path is infinite, set $\alpha:=\omega$, and let $f(c)$ be the biggest $i \in \omega$ such that $c \in Z_{i}$. Since $\bigcap_{i \in \omega} Z_{i}=\emptyset$ this is well-defined.

(2) If the Zielonka path is finite and does not end with the empty set, let $\alpha:=\beta$ and define $f(c)$ as in the first case.

(3) If the Zielonka path is finite, and ends with $Z_{2 j+2}=\emptyset$, let $\alpha=\omega$ and define $f: C \rightarrow \omega$ as follows. For $i<2 j+1$ we put $f(c)=i$ for all $c \in Z_{i} \backslash Z_{i+1}$. For $c \in Z_{2 j+1}$ we define $f(c)$ by means of a bijection from the (infinite) set $Z_{2 j+1}$ to the set of yet unused odd priorities $\{2 n+1: n \geq j\}$.

Cleary, for any non-empty $X$, we have that $X \in \mathcal{F}_{0}$ if, and only if, $\min f(X)$ is even. Further, $f^{-1}(i)$ is infinite only in the case that $f^{-1}(i)=Z_{i}$ is the last set in the Zielonka path. In that case $i$ is even and is the maximal element in the range of $f$. Hence $f$ defines an appropriate reduction from $\left(\mathcal{F}_{0}, \mathcal{F}_{1}\right)$ to a parity condition.

We can now summarize the characterisation of the Muller conditions that guarantee positional determinacy.

Theorem 5.12. For any Muller condition $\left(\mathcal{F}_{0}, \mathcal{F}_{1}\right)$ over a countable set $C$ of priorities, the following are equivalent.

(1) $\left(\mathcal{F}_{0}, \mathcal{F}_{1}\right)$ guarantees positional winning strategies. 
(2) $\mathcal{F}_{0}$ and $\mathcal{F}_{1}$ are closed under union of chains, non-empty intersections of chains, and have no strong splits.

(3) $\left(\mathcal{F}_{0}, \mathcal{F}_{1}\right)$ is described by a Zielonka path of co-finite sets.

(4) $\left(\mathcal{F}_{0}, \mathcal{F}_{1}\right)$ reduces to a parity condition on an ordinal $\alpha \leq \omega$.

Determinacy of Muller Games. Theorem 5.12 classifies the Muller conditions that imply positional determinacy on all game graphs. We remark that for Muller games, determinacy itself is an issue that deserves investigation. If either $\mathcal{F}_{0}$ or $\mathcal{F}_{1}$ is countable, then the Muller condition $\left(\mathcal{F}_{0}, \mathcal{F}_{1}\right)$ is Borel (on level $\boldsymbol{\Sigma}_{4}^{0}$ or $\boldsymbol{\Pi}_{4}^{0}$ ), so determinacy follows from Martin's Theorem. In general however, Muller conditions over countable sets of priorities need not be Borel. This can be seen via a simple counting argument. There are only $2^{\aleph_{0}}$ Borel sets since each of them is described by a countable infinitary formula. But there are $2^{2^{\aleph_{0}}}$ Muller conditions. Indeed, on the basis of Boolean Prime Ideal Theorem, which is a weak form of the Axiom of Choice, it is not too difficult to construct non-determined Muller games.

Theorem 5.13. There exist non-determined infinitary Muller games.

Proof. We slightly modify a well-known construction of a non-determined Gale-Stewart game. The Boolean Prime Ideal Theorem implies that there exists a free ultrafilten $U \subseteq$ $\mathcal{P}(\omega)$. Let $\mathcal{F}_{0}=U$ and construct a game graph such that by playing the game, the players define a strictly increasing sequence $a_{0}<a_{1}<a_{2}<\ldots$, where the numbers $a_{2 n}$ are chosen by Player 0 , and numbers $a_{2 n+1}$ by Player 1 , such that precisely the priorities in $X:=\bigcup_{n \in \omega}\left(a_{2 n}, a_{2 n+1}\right]$ are seen infinitely often.

We claim that the resulting Muller game is not determined. Assume that Player 0 has a winning strategy $f$ which maps any increasing sequence $a_{0}<a_{1}<\cdots<a_{2 n-1}$ of even length to $a_{2 n}=f\left(a_{0} a_{1} \ldots a_{2 n-1}\right)>a_{2 n-1}$. We consider two intertwined counter-strategies of Player 1, forcing essentially Player 0 to simultaneously perform two plays against himself. In reply to the first move $a_{0}$, Player 1 selects an arbitrary $a_{1}>a_{0}$ and then sets up the two plays as follows: In the first one she replies to $a_{0}$ by $a_{1}$ and waits for the answer $a_{2}=f\left(a_{0} a_{1}\right)$ by Player 0 . She then uses $a_{2}$ as her own reply to $a_{0}$ in the second play and gets the answer $a_{3}=f\left(a_{0} a_{2}\right)$ by Player 0 , which she now uses as her next move in the first play. There Player 0 responds by $a_{4}=f\left(a_{0} a_{1} a_{2} a_{3}\right)$ which is again used by Player 1 as her answer to $a_{0} a_{2} a_{3}$ in the second play. And so on.

In this way, the two infinite plays result in sequences $a_{0}<a_{1}<a_{2}<\ldots$ and $a_{0}<$ $a_{2}<a_{3}<\ldots$. Since Player 0 plays with his winning strategy in both plays. it follows that $X=\bigcup_{n \in \omega}\left(a_{2 n}, a_{2 n+1}\right] \in U$, but also $X^{\prime}=\left(a_{0}, a_{2}\right] \cup \bigcup_{n>0}\left(a_{2 n+1}, a_{2 n+2}\right] \in U$. By closure under intersection, it follows that $X \cap X^{\prime}=\left(a_{0}, a_{1}\right] \in U$. But $U$ is a free ultrafilter, so it cannot contain a finite set.

By almost precisely the same argument, it also follows that Player 1 cannot have a winning strategy.

\footnotetext{
${ }^{1}$ An ultrafilter in $\langle\mathcal{P}(\omega), \subseteq\rangle$ is a set $U \subseteq \mathcal{P}(\omega)$ that does not contain $\emptyset$, that includes with any set also all its supersets, with any two sets also their intersection, and such that for any set $x \subseteq \omega$ either $x \in U$ or $\omega \backslash x \in U$. An ultrafilter is free if it contains all co-finite sets. As a consequence, it does not contain any finite set.
} 


\section{Further ReSUlts}

6.1. Uncountable sets of priorities. In the previous section we have assumed that the set of all priorities is countable. However, it can be shown that the characterization of the Muller conditions that guarantee positional winning strategies remains the same for uncountable sets of priorities. As in each play, there appear only countably many priorities, uncountable sets play no role in a Muller condition. Still, the argument is slightly more involved than in Theorem 5.12 because we cannot start the construction from the set $C$ of all priorities. Nevertheless, we can show that if every restriction of the Muller condition to a countable subset of $C$ satisfies (P0), (P1), (P2) then it is equivalent to a parity condition.

Let $\left(\mathcal{F}_{0}, \mathcal{F}_{1}\right)$ be a Muller condition over an uncountable set $C$ of priorities.

Definition 6.1. A set $X \in \mathcal{F}_{0}$ is called a 0 -limit set if whenever $X \subseteq X^{\prime}$ then $X^{\prime} \in \mathcal{F}_{0}$. Similarly for 1-limit sets.

Lemma 6.2. There exists a countable 0 or 1-limit set.

Proof. If not then we can construct an infinite increasing sequence of countable sets such that even indexed sets are from $\mathcal{F}_{0}$ and odd indexed sets are from $\mathcal{F}_{1}$.

Lemma 6.3. If there is a 0-limit set then there is no 1-limit set.

Proof. Otherwise there is a 0 -limit set $X$ and a 1 -limit set $Y$. Consider $X \cup Y$. By definition of limit sets it should belong to both $\mathcal{F}_{0}$ and $\mathcal{F}_{1}$.

Lemma 6.4. Suppose that $Y \in \mathcal{F}_{1}$ and that $Y \cup\{a\} \in \mathcal{F}_{0}$ is a 0 -limit set. In this case $\{a\}$ is a 0 -limit set.

Proof. Assume conversely that there is a $Y^{\prime} \in \mathcal{F}_{1}$ containing a. We have $Y \cup Y^{\prime}=Y \cup Y^{\prime} \cup$ $\{a\} \in \mathcal{F}_{0}$ by the assumption that $Y \cup\{a\}$ is a limit set. Let $Y_{1}$ be the greatest element of $\mathcal{F}_{1}$ included in $Y \cup Y^{\prime}$; it exists by construction from Thm 5.12, As $Y, Y^{\prime} \in \mathcal{F}_{1}$, we have then $Y \subseteq Y_{1}$ and $Y^{\prime} \subseteq Y_{1}$, but this is impossible as it implies that $Y_{1}=Y \cup Y^{\prime} \in \mathcal{F}_{0} \cap \mathcal{F}_{1}=\emptyset$.

Lemma 6.5. For every limit set $X$ there is a priority $a \in X$ such that $\{a\}$ is a limit set.

Proof. Take a limit set $X \in \mathcal{F}_{0}$ and the greatest set $Y \subseteq X$ from $\mathcal{F}_{1}$. The set $D:=X \backslash Y$ is finite by the construction from Thm 5.12. Take an arbitrary element $a \in D$. If $Y \cup\{a\}$ is a 0 -limit set then we are done by the previous lemma. If not then we take $Y^{\prime} \in \mathcal{F}_{1}$ containing $Y \cup\{a\}$. Then we take a next priority $b \in D$ and consider $Y^{\prime} \cup\{b\}$. As $D$ is finite and $Y \cup D=X$ is a limit set we can repeat these steps at most $|D|$ number of times.

The above lemma allows to introduce the notion of a limit priority. Let us remove all the limit priorities from $C$ and consider the Muller condition $\left(\mathcal{F}_{0}^{\prime}, \mathcal{F}_{1}^{\prime}\right)$ obtained by restricting $\left(\mathcal{F}_{0}, \mathcal{F}_{1}\right)$ to this set of priorities. Clearly it also satisfies the conditions P1, P2, P3.

Lemma 6.6. In $\left(\mathcal{F}_{0}^{\prime}, \mathcal{F}_{1}^{\prime}\right)$ there is no limit set from $\mathcal{F}_{0}^{\prime}$.

Proof. If there were a limit set in $\mathcal{F}_{0}^{\prime}$ then this set would be also a limit set with respect to the original condition $\left(\mathcal{F}_{0}, \mathcal{F}_{1}\right)$. But then it would contain a limit priority which is impossible by the definition of $\left(\mathcal{F}_{0}^{\prime}, \mathcal{F}_{1}^{\prime}\right)$. 
Hence in $\left(\mathcal{F}_{0}^{\prime}, \mathcal{F}_{1}^{\prime}\right)$ there is a limit set in $\mathcal{F}_{1}$ and we can choose limit priorities for the other player. Repeat the construction for $\omega$ steps. We obtain a sequence of nonempty sets of priorities $A_{1}, B_{1}, \ldots$ If after $\omega$ steps the set of remaining priorities is nonempty then we take a countable subset $R$ of the priorities that are left and have a decreasing sequence $R_{1}, R_{2}, \ldots$, where $R_{2 i-1}=\bigcup\left\{a_{i}, b_{i}, a_{i+1}, b_{i+1}, \ldots\right\} \cup R$ and $R_{2 i}=\bigcup\left\{b_{i}, a_{i+1}, b_{i+1}, \ldots\right\} \cup R$. But the existence of such a sequence contradicts the condition P1.

6.2. Games of bounded degree. The question arises whether the class of winning conditions that guarantee positional winning strategies becomes larger if we only consider game graphs of finite degree, or game graphs of finite and bounded degree. In particular this question has been asked for max-parity games and for parity games over larger ordinals than $\omega$, where the counter-example that we have presented has infinite degree. It turns out that parity games over $\omega+1$ are determined, while those over $\omega+2$ are not. However, it seems quite difficult to give an exact characterisation of the Muller conditions that guarantee positional winning strategies on all game graphs of bounded finite degree.

Proposition 6.7. Max-parity games with infinitely many priorities in general do not admit finite memory strategies, even for solitaire games and even for game graphs with maximimal degree two.

Proof. Consider the following game where every vertex has degree one or two.

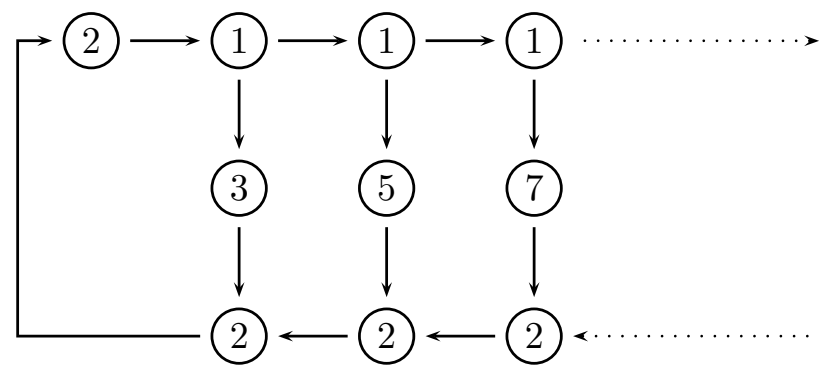

Assuming the max-parity winning condition it is obvious that there is an infinite memory strategy for Player 0 to enforce that the set of priorities seen infinitely often is $\{1,2\}$, but that any finite memory strategy is losing.

The same construction works for (min-)parity games on ordinals $\alpha>\omega+1$. Indeed, if we replace priorities 2 and 1 by $\omega$ and $\omega+1$, we obtain a min-parity game that requires an infinite memory winning strategy.

However there is an interesting case where parity games of bounded degree behave differently than games of unbounded degree.

Theorem 6.8. Parity games of bounded degree with priorities in $\omega+1$ are positionally determined.

Proof. We first consider the case of Player 1. Extending the argument from Theorem 4.3 we show that in any parity game with priorities in $\omega+1$, Player 1 has a positional winning strategy on his winning region. Note that for this case we do not need the assumption of bounded degree.

Let $\mathcal{G}$ a parity game with priorities in $\omega+1$, let $g$ be a winning strategy for Player 1 on his winning region in $\mathcal{G}$ and let $\mathcal{T}_{g}$ be the associated strategy forest. In the following, 
positions labeled by $\omega$ will be called $\omega$-positions and the other positions will be called natural positions. Since every path through $\mathcal{T}_{g}$ is winning for Player 1, it must contain infinitely many natural positions. This means that the definitions of 1-signatures that we have used in the proof for parity games on $\omega$ carry over here. Recall that $<_{i}^{1}$ denotes the signature order (which is strict and partial). Let $\triangleleft_{i}$ be a total well-order extending $<_{i}^{1}$, and assume that $\perp$ is the biggest element in this order.

As in the proof of Theorem 4.3 we associate with every node $s$ of $\mathcal{T}_{g}$ of priority $m<\omega$ the $(m+1)$-tuple $a(s)=\left\langle a_{0}(s), \ldots, a_{m}(s)\right\rangle \in\left(\mathcal{T}_{g} \cup\{\perp\}\right)^{m+1}$ of ancestors. As before, we write $s \prec_{m} s^{\prime}$ if there is $i<m$ such that $a_{i}(s) \triangleleft_{i} a_{i}\left(s^{\prime}\right)$ and $a_{j}(s)=a_{j}\left(s^{\prime}\right)$ for all $j<i$. Observe that $\prec_{m}$ is a well-order on vertices of priority $m$. For a position $v$ of priority $m$ we now take the $\prec_{m}$-minimal representant of $v$ in $\mathcal{T}_{g}$, i.e., the minimal $s$ with $h(s)=v$ where $h$ is the canonical homomorphism from $\mathcal{T}_{g}$ to $\mathcal{G}$. We denote this representant by $s(v)$. We can also define the tuple of ancestors by $a(v)=a(s(v))$.

In order to define $s(v)$ for positions of priority $\omega$ we use $\prec_{m}$ to compare vertices in $\mathcal{T}_{g}$ of different, but finite, priority. We define $s \prec s^{\prime}$ if either $s \prec_{m} s^{\prime}$ for $m$ being the minimum of the priorities of $s$ and $s^{\prime}$, or the tuple $\left(a_{0}(s), \ldots, a_{m}(s)\right)$ has $\left(a_{0}\left(s^{\prime}\right), \ldots, a_{m^{\prime}}\left(s^{\prime}\right)\right)$ as a strict prefix. We claim that this is a well-ordering on vertices of finite priority. To reason by contradiction, suppose that there is an infinite descending chain $s_{0} \succ s_{1} \succ \ldots$ in this ordering. Let us look at the chain $a_{0}\left(s_{0}\right), a_{0}\left(s_{1}\right), \ldots$ of first elements of the tuples. This chain is not increasing in the $\triangleleft_{0}$-ordering, so it must eventually stabilise on some element $a_{0}$. Let $i_{0}$ be the position where it stabilises. Observe that this implies that there cannot be a vertex of priority 0 after $s_{0}$. By a similar argument we find $a_{1}$ that stabilizes after $a_{0}$ stabilizes. Continuing like this we get an infinite sequence $a_{0}, a_{1}, \ldots$ Observe that infinitely many of the elements in the sequence are not $\perp$. Indeed, there are vertices of infinitely many priorities, and when we see a vertex $s$ of priority $i$ then $a_{i}(s)$ is not $\perp$ so $a_{i}$ cannot be $\perp$. To finish the argument we observe that for each $i=0,1, \ldots$, if $a_{i} \neq \perp$ then it is a vertex of priority $i$ and it is an ancestor of all $a_{j} \neq \perp$ for $j>i$. Moreover, there can be no vertices of priority $i$ between $a_{i}$ and $a_{j} \neq \perp$ for $j>i$. Thus, the sequence $a_{0}, a_{1}, \ldots$ determines an infinite path in $\mathcal{T}_{g}$ where no priority, except possibly $\omega$, appears infinitely often. This is a contradiction as we have assumed that all paths in $\mathcal{T}_{g}$ are winning for Player 1.

There are two more notions that we need. For each vertex $s \in \mathcal{T}_{g}$ of priority $\omega$ we define the max-distance to be the maximal length of a path of $\omega$-vertices starting from $s$. This is well defined as on every path from $s$ there is eventually a vertex of a finite priority. Secondly, for $s$ we define its anchor to be the closest ancestor of finite priority.

Now we are ready to define $s(v)$ for positions $v$ of priority $\omega$. Among all the representants of $v$, i.e., vertices $s$ such that $h(s)=v$, we choose one with the $\prec$-smallest anchor. If there are more than one with this property then among them we choose the one with the smallest max-distance. If this still does not identify a unique representant then we choose one arbitrarily.

Having defined $s(v)$ for all $v$ in the winning region for Player 1 in $\mathcal{G}$ we define a positional strategy $g^{\prime}$. We set $g^{\prime}(v)=h(t)$ where $t$ is the unique successor of $s(v)$ in $\mathcal{T}_{g}$. We claim that this strategy is winning. Suppose conversely that there is a loosing play respecting the strategy. Then either no natural number appears infinitely often or the smallest number appearing infinitely often is even.

If no natural number appears infinitely often then we proceed as in the proof of Theorem 3.2. Consider the suffix of the play after the last appearance of priority 0. Let us look at 0 -ancestors of the positions in this suffix. These ancestors can only get smaller as 
the play proceeds. This means that from some moment all positions in the play will have the same 0 -ancestor. Next, we find a position where the 1-ancestor stabilizes. Observe that it will be a descendant of the 0 -ancestor and that there will be no occurrences of priority 0 between the two. Proceeding this way we construct a path in the strategy tree $\mathcal{T}_{g}$ on which no priority, accept possibly $\omega$, appears on infinite number of times. Notice, that it is important for this argument that the sequences of $\omega$-nodes are finite. The remaining case when the smallest number appearing infinitely often is even is very similar to that from the proof of Theorem 4.3.

To show that Player 0 can win with a positional strategy we transform a game $\mathcal{G}$ with priorities from $\omega+1$ into a game $\widehat{\mathcal{G}}$ and then to $\widetilde{\mathcal{G}}$, such that $\widetilde{\mathcal{G}}$ has no $\omega$-positions. Then we translate the positional winning strategy form $\widetilde{\mathcal{G}}$ to $\mathcal{G}$.

We first describe the transformation from $\mathcal{G}$ to $\widehat{\mathcal{G}}$. Take a position $s$ of $\mathcal{G}$ labeled with $\omega$. For any $i \in \omega \cup\{\omega\}$ consider a gadget $K_{s}^{i}$ :

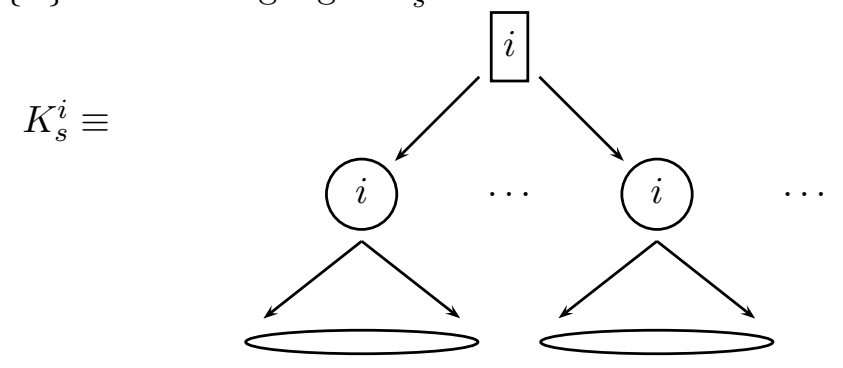

Each round vertex represents a strategy of Player 1 from $s$ permitting him to leave the region of $\omega$-labeled positions. The oval below such a vertex represents the possible exits, i.e., the natural positions that Player 0 can reach when Player 1 uses the chosen strategy. Observe that if Player 0 has a strategy to stay in $\omega$-positions then the root of $K_{s}^{\omega}$ has no successors. To be conform with our definition of the game, in this case we assign a priority 0 to the root of $K_{s}^{\omega}$ and add a self-loop. This way we make it winning for Player 0. We call such a gadget degenerate.

The transformation from $\mathcal{G}$ to $\widehat{\mathcal{G}}$ is the following. Take an $\omega$-position $s$ and replace it by the gadget $K_{s}^{\omega}$. The leaves of this gadget are natural positions in $\mathcal{G}$, hence we only add one position of Player 1 and some positions of Player 0. Redirect every arrow going from a natural position to $s$ to the root of $K_{s}^{\omega}$. Repeating this for all $\omega$-positions (of the game $\mathcal{G}$ ) we obtain the game $\widehat{\mathcal{G}}$. This game has the property that sequences of $\omega$-vertices can have length at most 2. Moreover there is an easy correspondence between strategies in $\mathcal{G}$ and $\widehat{\mathcal{G}}$.

Next we describe the transformation from $\widehat{\mathcal{G}}$ to $\widetilde{\mathcal{G}}$. The idea is to eliminate the priorities $\omega$. If we come to a gadget from a position of priority $i$ then we can as well assume that we see $i$ in place of $\omega$. The result of an infinite play will be the same as we at most triple the number of $i$ 's seen. For example, if $\omega$ was the only priority appearing infinitely often then after the change no priority at all would appear infinitely often, which gives the win to the same player. The transformation from $\widehat{\mathcal{G}}$ to $\widetilde{\mathcal{G}}$ is as follows. For each priority $i \in \omega$ and each non-degenerate gadget $K_{s}^{\omega}$ we create a gadget $K_{s}^{i}$, which has priority $\omega$ replaced by the priority $i$. For each position $u$ of priority $i$ in $\widehat{\mathcal{G}}$ with an arrow to the root of $K_{s}^{\omega}$ we redirect this arrow to the root of $K_{s}^{i}$. Of course we need not to create $K_{s}^{i}$ if there are no such $u$. Repeating this procedure for each gadget $K_{s}^{\omega}$ we get rid of all positions of priority $\omega$. The result is the game $\widetilde{\mathcal{G}}$. 
There is a canonical homomorphism $\widetilde{h}: \widetilde{\mathcal{G}} \rightarrow \widehat{\mathcal{G}}$ which maps the root of $K_{s}^{i}$ to the root of $K_{s}^{\omega}$. It should be clear that there is a winning strategy in $\widetilde{G}$ if, and only if, there is one in $\widehat{G}$ (the image of a path is winning for Player 0 if, and only if, the path is a winning play for Player 0$)$. As in $\widetilde{G}$ there are no vertices of priority $\omega$ we know that Player 0 has a positional winning strategy on his winning region. We will show how to translate it into a positional winning strategy in $G$ (using $\widehat{G}$ on the way).

Take a positional winning strategy $f$ for Player 0 in $\widetilde{G}$. Consider the signature assignment in $\widetilde{G}$ defined by the strategy (as described in the section on parity games). This defines a signature assignment on natural positions of $\mathcal{G}$. It remains to define signatures for $\omega$-positions and then use it to define a winning strategy. For each $\omega$-position $s$ consider the gadget $K_{s}^{i}$ for some $i$. If the gadget is degenerated then in $\mathcal{G}$ Player 0 has a strategy from $s$ to stay in $\omega$-positions. We are done in this case as we can assume that Player 0 has one global positional strategy on all vertices with this property. We call such $s$ immediately winning. Suppose then that $K_{s}^{i}$ is not degenerate and $f$ is winning from its root. Each leaf of the gadget has assigned a signature. We can define the signature of $s$, denoted also by $\operatorname{sig}^{0}(s)$, by taking inf in nodes of Player 0 in $K_{s}^{i}$ and then sup in $s$. Here inf and sup are in the lattice of $\omega$-vectors of ordinals. Observe that $\operatorname{sig}^{0}(s)$ does not depend on the choice of $i$ in $K_{s}^{i}$. In order to have a uniform notation let $\leq_{\omega}^{0}$ denote the standard lexicographic ordering on $\omega$-tuples of ordinals. Notice that this is not a well-order while $\leq_{i}^{0}$ for $i \in \omega$ are. With this definition of signatures we have that if $s$ is a position of Player 1 , then for every successor $t$ that is not immediately winning we have $\operatorname{sig}(t) \leq_{i}^{0} \operatorname{sig}(s)$ where $i$ is a priority of $s$. Similarly, if $s$ is a position of Player 0 , then it has a successor $t$ which is either immediately winning or satisfies the same property. Having this property we can define a positional strategy for Player 0 that consists of choosing the smallest possible signature. The proof that this strategy is winning is the same as in the case of parity games.

This theorem indicates that when we limit ourselves to game graphs of finite degree the class of Muller conditions guaranteeing positional winning strategies becomes larger. There also exist Muller conditions that do not reduce to parity conditions over any ordinal but still guarantee positional winning strategies on all game graphs of finite degree. For finite sets of priorities, such examples are well-known. In the simplest one, the set of priorities is $C=\{0,1\}$, with $\mathcal{F}_{0}=\{\{0,1\}\}$ and $\mathcal{F}_{1}=\{\{0\},\{1\}\}$.

Similar examples with an infinite set of colours can be constructed as follows. Let $Y$ be any infinite set with $e \notin Y$ and set $C=Y \cup\{e\}$. Put

$$
\mathcal{F}_{0}=\mathcal{P}(Y) \cup\{\{e\}\} \cup\{\emptyset\} \quad \mathcal{F}_{1}=\{Z: e \in Z \wedge Z \cap Y \neq \emptyset\}
$$

It should be clear that $\left(\mathcal{F}_{0}, \mathcal{F}_{1}\right)$ is not equivalent to a parity condition because each priority individually is winning for Player 0. By arguments that are similar to the proof of Theorem 6.8 one can show that such a condition guarantees positional determinacy on all game graphs of finite degree.

It is an open problem to give a complete characterisation of all such conditions.

6.3. Finite appearance of priorities. We may also ask whether the characterisation of positionally determined Muller conditions changes if we only consider games where $\Omega^{-1}(c)$ is finite for every priority $c$. This is not the case. Indeed, the counter-examples for properties (P0) and (P2) are games with this property, and in the counter-example for (P1) we can easily eliminate infinite occurrences of priorities. Consider the figure in the proof of 
Lemma 5.3. It suffices to omit the sets $X_{2}, X_{3}, \ldots$ and redirect, for every $i \geq 2$, each arrow from $a$ to an element $x_{i} \in X_{i}$ to the element $x_{i} \in X_{1}$.

6.4. Related work. There has recently been some interesting research on similar questions for games in somewhat different settings. For instance, Colcombet and Niwiński [8] have studied positional determinacy of games where edges, rather than vertices are labeled by priorities. This changes the situation completely. For instance, it is easily seen that there are edge-labeled parity games with infinitely many priorities that require winning strategies with infinite memory. Also there are some very simple non-Muller winning conditions that guarantee positional determinacy on vertex-labeled games but fail to do so on edge-labeled ones. An example is the set $(0+1)^{*}(01)^{\omega}$ whare Player 0 has to make sure that from some point onwards the priorities 0 and 1 alternate. If she can achieve this on a vertex-labelled game then she can also do this positionally. However, when the priorities are on the edges, then this is not the case: consider the game with a single vertex and two self-loops with priorities 0 and 1 . In fact, Colcombet and Niwiński prove that the only prefix-independent winning conditions that guarantee positional determinacy on all edge-labeled game graphs are precisely the parity conditions with a finite number of priorities. In a similar vein, Kopczynski [20] characterises the winning conditions that guarantee positional determinacy for one player on edge-labeled game graphs.

Serre [27] exhibits examples of winning conditions on a countable set of priorities that have high Borel complexity, but still admit positional winning strategies. Recall that in our setting, if the set of priorities is countable then the conditions are at most at levels $\Sigma_{4}^{0}$ or $\Pi_{4}^{0}$ of the Borel hierarchy.

Gimbert and Zielonka [13] consider edge-labeled games with real valued pay-offs. They characterise those pay-off functions that guarantee optimal positional strategies for both players on all finite game graphs. As in the case studied by Colcombet and Niwiński the payoffs are on edges and not on vertices.

\section{REFERENCES}

[1] A. Arnold, A. Vincent, And I. Walukiewicz, Games for synthesis of controllers with partial observation, Theoretical Computer Science, 303 (2003), pp. 7-34.

[2] D. Berwanger, A. Dawar, P. Hunter, And S. Kreutzer, Dag-width and parity games, in Proceedings of 23rd Annual Symposium on Theoretical Aspects of Computer Science, STACS 2006, Lecture Notes in Computer Science Nr. 3848, 2006, pp. 524-536.

[3] D. Berwanger And E. Grädel, Fixed-point logics and solitaire games, Theory of Computing Systems, 37 (2004), pp. 675-694.

[4] D. Berwanger And E. GRÄDEL, Entanglement - A measure for the complexity of directed graphs with applications to logic and games, in Proceedings of LPAR 2004, Lecture Notes in Computer Science Nr. 3452, Springer-Verlag, 2005, pp. 209-223.

[5] A. Blumensath and E. Grädel, Finite presentations of infinite structures: Automata and interpretations, Theory of Computing Systems, 37 (2004), pp. $641-674$.

[6] A. Bouquet, O. Serre, And I. Walukiewicz, Pushdown games with unboundedness and regular conditions, in Proceedings of FSTTCS'03, Lecture Notes in Computer Science Nr. 2914, 2003, pp. 8899.

[7] T. Cachat, J. Duparc, And W. Thomas, Solving pushdown games with a $\Sigma_{3}$ winning cndition, in Computer Science Logic, CSL 2002, Lecture Notes in Computer Science Nr. 2471, Springer-Verlag, 2002, pp. 322-336.

[8] T. Colcombet And D. Niwiński, On the positional determinacy of edge-labeled games, Theoretical Computer Science, (2006). 
[9] S. Dziembowski, M. Jurdziński, And I. Walukiewicz, How much memory is needed to win infinite games?, in Proceedings of 12th Annual IEEE Symposium on Logic in Computer Science (LICS 97), 1997, pp. 99-110.

[10] A. Emerson And C. Jutla, Tree automata, mu-calculus and determinacy, in Proc. 32nd IEEE Symp. on Foundations of Computer Science, 1991, pp. 368-377.

[11] A. Emerson, C. Jutla, And P. Sistla, On model checking for the $\mu$-calculus and its fragments, Theoretical Computer Science, 258 (2001), pp. 491-522.

[12] H. Gimbert, Parity and exploration games on infinite graphs, in Proceedings of CSL 2004, Lecture Notes in Computer Science Nr. 3210, Springer, 2004, pp. 56-70.

[13] H. Gimbert And W. ZielonkA, Games where you can play optimally without any memory, in CONCUR 2005 - Concurrency Theory, 16th International Conference, Lecture Notes in Computer Science Nr. 3653, Springer, 2005, pp. 428-442.

[14] E. GräDEL, Finite Model Theory and Descriptive Complexity, in Finite Model Theory and Its Applications, Springer-Verlag, 2006. To appear.

[15] E. Grädel, W. Thomas, and T. Wilke, eds., Automata, Logics, and Infinite Games, Lecture Notes in Computer Science Nr. 2500, Springer, 2002.

[16] Y. Gurevich and L. Harrington, Trees, automata and games, in Proceedings of the 14th Annual ACM Symposium on Theory of Computing, STOC '82, 1982, pp. 60-65.

[17] M. JuRdziński, Deciding the winner in parity games is in $U P \cap C o-U P$, Information Processing Letters, 68 (1998), pp. 119-124.

[18] M. JuRdziński, Small progress measures for solving parity games, in Proceedings of 17th Annual Symposium on Theoretical Aspects of Computer Science, STACS 2000, Lecture Notes in Computer Science Nr. 1770, Springer, 2000, pp. 290-301.

[19] M. Jurdziński, M. Paterson, And U. Zwick, A deterministic subexponential algorithm for solving parity games, in Proceedings of ACM-SIAM Proceedings on Discrete Algorithms, SODA 2006, 2006, pp. 117-123.

[20] E. Kopczynski, Half-positional determinacy of infinite games, in Automata, Languages and Programming, 33rd International Colloquium, ICALP 2006, Lecture Notes in Computer Science Nr. 4052, 2006, pp. 336-347.

[21] O. KupfERMAn AND M. VARDI, An automata-theoretic approach to reasoning about infinite-state systems, in Proceedings of 12th International Conference on Computer-Aided Verification CAV 2000, Lecture Notes in Computer Science Nr. 1855, Springer, 2000, pp. 36-52.

[22] D. Martin, Borel determinacy, Annals of Mathematics, 102 (1975), pp. 336-371.

[23] R. McNaughton, Infinite games played on finite graphs, Annals of Pure and Applied Logic, 65 (1993), pp. $149-184$.

[24] A. Mostowski, Games with forbidden positions, Tech. Rep. Tech. Report 78, University of Gdansk, 1991.

[25] J. OBdRZAleK, Fast mu-calculus model checking when tree-width is bounded, in Proceedings of CAV 2003, vol. 2752 of LNCS, Springer, 2003, pp. 80-92.

[26] J. OBDRZALEK, DAG-width - connectivity measure for directed graphs, in Proceedings of ACM-SIAM Proceedings on Discrete Algorithms, SODA 2006, 2006, pp. 814-821.

[27] O. SERRE, Games with winning conditions of high Borel complexity, in Proceedings of ICALP 2004, vol. 3142 of Lecture Notes in Computer Science, 2004, pp. 1150-1162.

[28] I. Walukiewicz, Pushdown processes: Games and model checking, Information and Computation, 164 (2001), pp. 234-263.

[29] W. ZielonKA, Infinite games on finitely coloured graphs with applications to automata on infinite trees, Theoretical Computer Science, 200 (1998), pp. 135-183.

This work is licensed under the Creative Commons Attribution-NoDerivs License. To view a copy of this license, visit http://creativecommons.org/licenses/by-nd/2.0/ or send a letter to Creative Commons, 559 Nathan Abbott Way, Stanford, California 94305, USA. 